\title{
Influence of the Heating Processes on the Modal Attenuation Distribution in Planar Gradient Waveguides
}

\author{
T. KOTYCZKA ${ }^{a}$ AND R. ROGOZIŃSKI ${ }^{b, *}$ \\ ${ }^{a}$ WASKO S.A., Berbeckiego 6, 44-100 Gliwice, Poland \\ ${ }^{b}$ Department of Optoelectronics, Silesian University of Technology, B. Krzywoustego 2, 44-100 Gliwice, Poland \\ In this paper the influence of the heating processes on modal attenuation distribution in planar gradient \\ waveguides produced by ion exchange method in soda-lime glass $-\mathrm{Ag}^{+}$admixture arrangement is presented. The \\ influence of heating processes on modal attenuation distribution in dependence on preliminary refractive profile \\ shape was analysed. Numerical simulations were based on experimentally gained thermal diffusion coefficients of \\ the exchanged ions.
}

PACS: 81.20.Fw, 42.70.Ce, 07.07.Df

\section{Introduction}

Nowadays research on chemical environment sensors is an up-to-date topic, where there is still wide range of possible implementations, particularly if we consider planar technologies. Attractiveness of this kind of sensors is their operational simplicity which is based on waveguide mode suppression phenomenon. This effect occurs in mode's evanescent field interaction with the surroundings of an optical fibre. It could be considered in two ways: through the direct contact of waveguide's surroundings (change of its absorption) with the structure itself or in more indirect way through the absorption changes of the sensor layer covering the optical waveguide [1-3]. This kind of sensor structures can be constructed on optical fibres. Such solution facilitates connection of the sensor with optical track, however necessity of fibre core exposure for possible modal field influence with the surroundings is difficult. Lack of light sources in ranges of the largest absorption changes of ambient (the sensor layer) under influence of examined factor is an additional difficulty. The multimode optical fibre usage which can effectively cooperate with LED sources does not solve this problem. The possibility of modal coupling in such fibre as well as stimulating method causes that the amplitude changes of signal at the end of the structure could be dependent on power distribution between modes. Main goal therefore is to obtain a refractive index profile for which modal attenuation coefficients of the all mode orders are equal [4]. A vast possibility of waveguide's refractive profile shaping is to apply the ion exchange method where their shape is determined by mutual relationship among the diffusion coefficients of exchanged ions. Therefore the main shape determining factor of a refractive index

* corresponding author; e-mail: roman.rogozinski@polsl.pl profile is glass-admixture configuration. The concentration level of the source of admixture for a specific glass-admixture arrangement is also very important [5-7]. Additionally, there is a possibility of applying secondary processes, such as heating and diffusion processes or electrodiffusion, which allow us for even greater changes in shape of waveguide's refractive index profile [8, 9]. Apart from time and temperature of the mentioned processes with the increase of possible methods, the quantity of technological parameters that one should take into account during the profile modelling will also increase. In this work the main focus was placed on the basic type of preliminary diffusion as well as heating process, by which shape of the refractive profile could be repeatedly modelled. As mentioned, the main goal of these investigations was to show the influence of heating process on the shape of refractive profile hence the modal attenuation distribution of the specific waveguide. Real conditions of the applied technological processes were taken into account during this study. All initial refractive profiles of the planar waveguides subjected to heating processes were appointed based on structures realized in diffusion or electrodiffusion methods. Numerical simulations were based on experimentally gained thermal dependences of diffusion coefficients in exchanged ions. Therefore received results refer to real possibilities of the method used during this study.

The arrangement of this paper is as follows. In Sect. 2 the concept idea of planar multimode optical sensor was briefly introduced. Section 3 contains research assumptions and groundwork for numerical modelling of the technological processes. In Sect. 4 the calculation method which was used to designate modal attenuation coefficients was described. Section 5 contains research results. Summary and conclusions are gathered in Sect. 6 . 


\section{Multimode planar amplitude sensors}

The idea behind the operational technique of amplitude sensor based on multimode planar waveguide is the interaction of evanescent modal field with ambient or with the surface covering layer of the optical structure $[6,10]$ (Fig. 1). The suppression of modal fields depends on the depth of their penetration into the area where attenuation still exists (the surroundings or the sensor layer). In these considerations we assumed constant but small and stable modal attenuation in waveguide area. Additionally, with regard to a small length of such a structure (several $\mathrm{cm}$ ), lack of modal coupling phenomenon is assumed. In such a case assuming $P_{0}$ as the power inserted at the beginning of the waveguide $(z=0)$ which decomposes on individual modes, we can formulate such an equation

$$
P_{0}=\sum_{m=0}^{M-1} P_{m}(0)
$$

where $M$ is quantity of stimulated modes, $P_{m}(0)-$ optical power of the $m$-th mode at the beginning of the waveguide.

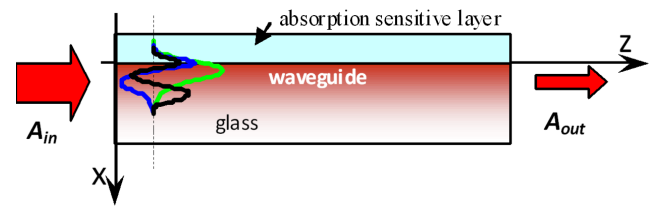

Fig. 1. Planar amplitude sensor structure.

Power distribution is a consequence of stimulating method at the beginning of the waveguide. With above assumptions after light propagation in accordance to the Lambert-Beer law, the total amount of power is described as

$$
P_{\text {tot }}(z, \gamma(m))=\sum_{m=0}^{M-1} P_{m}(0) \exp \left(-\gamma_{m} z\right) \text {. }
$$

In Eq. (2) $\gamma_{m}$ marks the attenuation coefficient of the $m$-th order mode. It is easy to notice that the initial power distribution $P_{m}(0)$ has the greatest impact on $P_{\text {tot }}(z, \gamma(m))$. Therefore amplitude modulation of $A_{\text {out }}$ signal at the end of structure is function of both $\gamma_{m}$ and $P_{m}(0)$. The constancy of $P_{m}(0)$ is difficult to achieve when the waveguide is stimulated by the multimode optical fibre. The solution for that is assurance of constancy of all the modal attenuation coefficients $\gamma_{m}=\gamma=$ const. Eqation (2) transforms then into

$$
P_{\text {tot }}(z, \gamma)=\exp (-\gamma z) \sum_{m=0}^{M-1} P_{m}(0) \text {. }
$$

In such case amplitude modulation of signal at the end of structure is entirely an effect of $\gamma$ absorption coefficients' changes being equal for every mode. Researches presented in this work are an attempt to achieve refrac- tive profile where changes of modal attenuation distribution will be as small as possible striving to the ideal case. They also allow us to determine trend in profile shape changes and at the same time let us point to best technological conditions.

\section{Research subject — process modelling}

The subject of the research were planar waveguides produced by ion exchange method $\mathrm{Ag}^{+} \leftrightarrow \mathrm{Na}^{+}$in soda-lime glass manufactured by Menzel-Gläser. The existence of homogeneous absorptive layer was assumed on waveguide's surface with the thickness of $d=100 \mathrm{~nm}$ and the real part of refractive index $n_{\mathrm{c}}^{(\mathrm{r})}=1.47$ [11] The modelling of waveguides refractive index profiles was based on experimentally calculated diffusion coefficients of the exchanged ions (Fig. 2) [5]. Based on the two-component ion exchange method mathematical model (Fig. 3) [5], the series of diffusion process simulations were conducted. Such model assumes dependence of diffusion coefficients $D_{\mathrm{A}}$ and the $D_{B}$ of exchanged ions on their normalised concentration

$$
\begin{aligned}
& D_{\mathrm{A}}(u)=D_{0 A} \mathrm{e}^{A u}, \\
& D_{B}(u)=D_{0 B} \mathrm{e}^{B(1-u)}, \\
& c_{0}=c_{\mathrm{A}}+c_{B}, \quad u=\frac{c_{\mathrm{A}}}{c_{0}}, \quad w=\frac{c_{B}}{c_{0}},
\end{aligned}
$$

where $u, w$ are normalised concentrations of admixture $\left(\mathrm{Ag}^{+}\right)$and the glass modifier $\left(\mathrm{Na}^{+}\right)$respectively, fulfilling the condition of $u+w=1$. The $D_{0 A}, D_{0 B}$ parameters are calculated experimentally. For planar structures the equation describing temporal-spatial changes of admixture concentration introduced to glass was shown as [5]:

$$
\begin{aligned}
& \frac{\partial u}{\partial t}=\frac{D_{0 A} \mathrm{e}^{A u}}{1-\alpha u} \frac{\partial^{2} u}{\partial x^{2}} \\
& +\frac{D_{0 A} \mathrm{e}^{A u}[\alpha+(1-u) A]-u(1-\alpha)^{2} D_{0 B} B \mathrm{e}^{B(1-u)}}{(1-\alpha u)^{2}} \\
& \quad \times\left(\frac{\partial u}{\partial x}\right)^{2} \\
& \alpha=1-\frac{D_{0 A}}{D_{0 B}} \mathrm{e}^{u(A+B)-B}
\end{aligned}
$$

Transition from solution $u(x, t)$ of Eq. (5) to refractive profile $n(x)$ takes place according to the following dependence:

$$
n(x)=n_{\mathrm{b}}+\Delta n u(x) .
$$

In the above mentioned equation $n_{\mathrm{b}}$ means the refraction index of the substrate. $\Delta n$ is, on the other hand, the maximum change of the refraction index near its surface. For calculation purposes wavelength of $\lambda=677 \mathrm{~nm}$, the TE light polarization as well as the refraction index of glass substrate with the value of $n_{\mathrm{b}}(677 \mathrm{~nm})=1.5105$ were assumed.

The main aspect however of the presented investigations is to show the influence of heating process on shape 


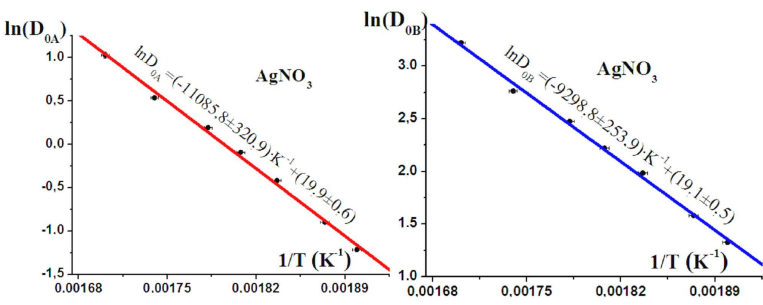

Fig. 2. Temperature dependences on diffusion coefficients $D_{0 A}$ and $D_{0 B}$ in soda-lime glass for pure source of admixture $\mathrm{AgNO}_{3}$.

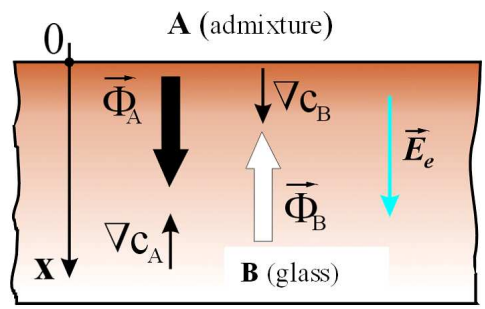

Fig. 3. Ion exchange process.

of refractive index profile. Presence of sensor layer produced with sol-gel method on the waveguide's surface requires execution of heating process in suitable temperature $[11,12]$. Therefore the heating processes applied to gradient waveguides obtained with ion exchange method will become mandatory processes.

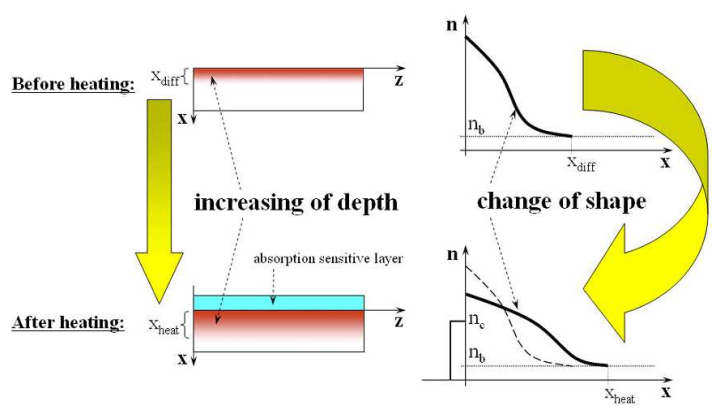

Fig. 4. Heating process' influence on refractive index profile shape.

Maintaining a planar structure in higher temperature causes major changes in shape of its refractive index profile. First of all, it increases the depth of the gradient structure which was received after diffusion process. Second change is significant decrease of the refraction index value near the glass surface. The influence of heating process is shown in Fig. 4. Effectiveness in refractive profile modeling of gradient waveguides as a result of heating process has an essential meaning for the numeric simulations conducted in next sections.

For credibility purposes of performed heating process simulations (Fig. 5a-c) demonstrates correlations between effective indexes obtained during measurements and those calculated from predicted refraction index profiles (Fig. 5d). All the cases are related to the same diffusion process from $\mathrm{AgNO}_{3}, T_{\text {diff }}=293^{\circ} \mathrm{C}, t_{\text {diff }}=3 \mathrm{~h}$. Temperatures of heating processes in each case are equal to $T_{\text {heat }}=293^{\circ} \mathrm{C}$. The heating times are $t_{\text {heat }}=15^{\prime}$, $t_{\text {heat }}=30^{\prime}$, and $t_{\text {heat }}=60^{\prime}$, respectively.

\section{Modal attenuation - calculations}

A calculation method described in this section is based on a formula which was deducted by Snyder and Love [13]:

$$
\gamma_{m}=2 k_{0} \sqrt{\frac{\varepsilon_{0}}{\mu_{0}}} \frac{\int_{-d}^{0} n_{\mathrm{c}}^{(\mathrm{r})} n_{\mathrm{c}}^{(\mathrm{i})}\left|\boldsymbol{E}_{m}\right|^{2} \mathrm{~d} x}{\operatorname{Re}\left(\int_{-\infty}^{\infty}\left(\boldsymbol{E}_{m} \times \boldsymbol{H}_{m}^{*}\right) \hat{e}_{z} \mathrm{~d} x\right)}
$$

where $\boldsymbol{E}_{m}, \boldsymbol{H}_{m}$ - the $m$-th mode electrical and magnetic field vectors. $k_{0}=2 \pi / \lambda_{0}$ - wave number in vacuum corresponding to wavelength $\lambda_{0} ; \varepsilon_{0}, \mu_{0}$ - permittivity and permeability in vacuum, respectively. The TE light polarization for calculations was accepted.

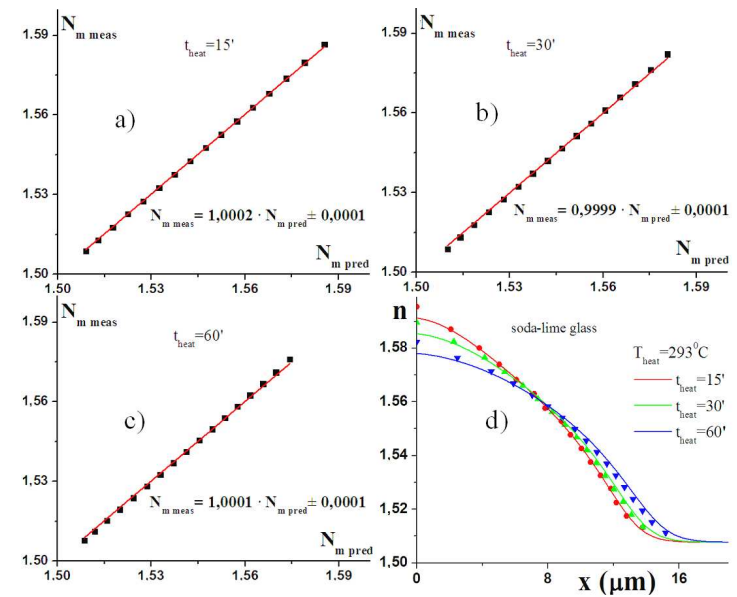

Fig. 5. Correlations between effective indexes obtained during measurements and those calculated from predicted refraction index profiles for the waveguides heated at $T_{\text {heat }}=293^{\circ} \mathrm{C}$ during (a) $t_{\text {heat }}=15^{\prime}$, (b) $t_{\text {heat }}=30^{\prime}$ and (c) $t_{\text {heat }}=60^{\prime}$, with corresponding refractive index profiles $(\mathrm{d})$.

It describes modal attenuation coefficients in a waveguide structure with the absorption area. The presented analysis assumes that on a gradient waveguide surface with the refraction index distribution described by $n(x)$ function, uniform absorptive layer with the refraction index $n_{\mathrm{c}}=n_{\mathrm{c}}^{(\mathrm{r})}-\mathrm{j} n_{\mathrm{c}}^{(\mathrm{i})}$ is deposited (Fig. 6a). In such a case with the arrangement shown in Fig. 6b Eq. (8) transforms to

$$
\gamma_{m}^{\mathrm{TE}}=\frac{2 k_{0} n_{\mathrm{c}}^{(\mathrm{r})} n_{\mathrm{c}}^{(\mathrm{i})}}{N_{m}^{\mathrm{TE}}} \frac{\int_{-d}^{0} E_{y, m}^{2}(x) \mathrm{d} x}{\int_{-\infty}^{\infty} E_{y, m}^{2}(x) \mathrm{d} x},
$$

where $N_{m}^{\mathrm{TE}}$ is $m$-th order mode's effective index. For practical reasons in presented deliberations the nor- 


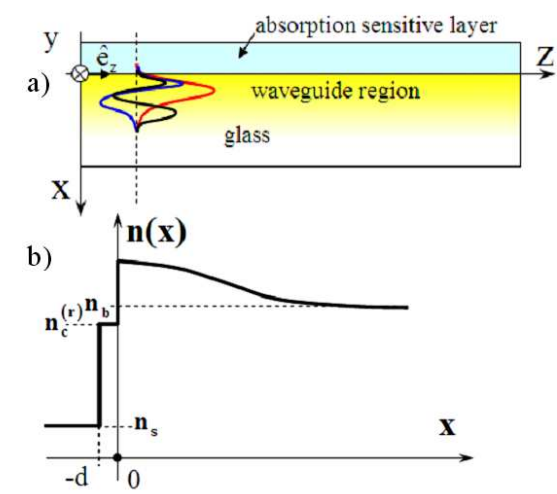

Fig. 6. Gradient waveguide with sensor layer (a), structure geometry (b).

malised modal attenuation coefficient $\tilde{\gamma}_{m}^{\mathrm{TE}}=\gamma_{m}^{\mathrm{TE}} / n_{\mathrm{c}}^{(\mathrm{i})}=$ $\tilde{\gamma}_{m}$ was accepted.

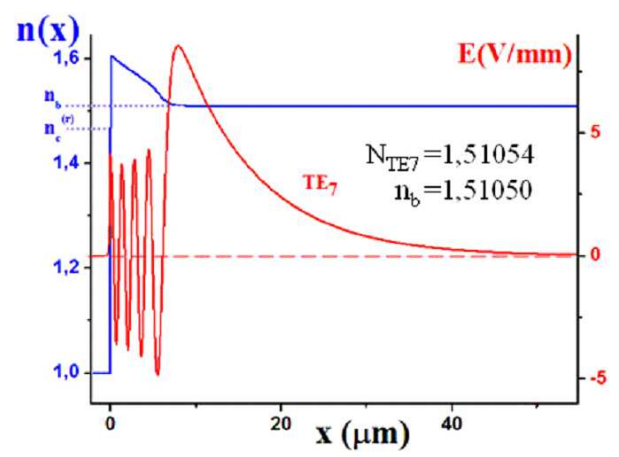

Fig. 7. Modal field of $\mathrm{TE}_{7}$ mode distribution near the cut-off area.

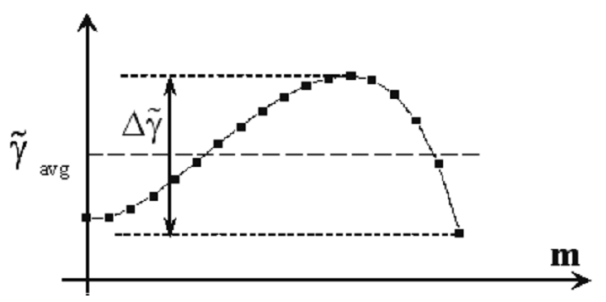

Fig. 8. Example of characteristic modal attenuation distribution.

The effective index calculations of propagating modes as well as the modal fields distribution for each waveguide structure were conducted with the use of matrix method $2 \times 2[14,15]$. In each case during research gradient refraction index area was divided into equally thick slices (monolayers). Taking into account the refractive profiles discretization influence on calculation results the equal division of gradient areas (800 monolayers) was accepted. Also in all the modal attenuation coefficient calculations the attenuation coefficients of the higher order modes were rejected. The reason for such a procedure is that rejected mode most often resides near the cut-off area. Therefore it is very difficult to stimulate it. At the same time the attenuation coefficient of such a mode is also very small. It results from the fact that the modal field which is located near the cut-off area penetrates deeply into the substrate material. Such behaviour was presented in Fig. 7. For a refractive index profile which was received during the diffusion process $\left(\mathrm{AgNO}{ }_{3}\right.$ source $)$ in the temperature $T_{\text {diff }}=300^{\circ} \mathrm{C}$ and process time $t_{\text {diff }}=50^{\prime}$, in presence of $n_{\mathrm{c}}=1.47$ layer with thickness $d=100 \mathrm{~nm}$, the $\mathrm{TE}_{7}$ mode is very close to the cut-off area and has refraction index larger about 0.00004 than the refraction index of the glass surface. So it is obvious that such a mode's field in large part passes to the substrate area. For further comparative analysis of modal attenuation distributions $\tilde{\gamma}(m)$ as a result of heating processes and in reference to different refractive profile mean values $\tilde{\gamma}_{\text {ave }}$ and partition spread in value changes $\Delta \tilde{\gamma}=\left(1-\tilde{\gamma}_{\min } / \tilde{\gamma}_{\max }\right) 100 \%$ were assumed. In Fig. 8 exemplary characteristic of modal attenuation distribution is presented.

\section{Influence of heating process on modal attenuation distribution}

\subsection{Refractive index profiles received during diffusion process}

The influence of heating process on a shape of refractive index profile of the waveguide produced with diffusion process is presented in Fig. 9a. It represents the shape of profile obtained during diffusion process of $\mathrm{Ag}^{+}$ ions (from pure silver nitrate $\mathrm{AgNO}_{3}$ as a source of admixture) to glass, where $T_{\text {diff }}=300^{\circ} \mathrm{C}, t_{\text {diff }}=300^{\prime}$. In the same figure calculated refractive index profiles of this waveguide after heating process in temperature $T_{\text {heat }}=300^{\circ} \mathrm{C}$ are introduced. It clearly shows the characteristic features of heating processes: decrease of refractive index near the waveguide's surface as well as depth increase of the gradient area with growth of the heating duration.

In Fig. 9b calculated characteristics of the modal attenuation distribution of planar waveguide for heating times $t_{\text {heat }}=10^{\prime}, 20^{\prime}, 40^{\prime}, 80^{\prime}$ and $160^{\prime}$ in the same temperature $T_{\text {heat }}=300^{\circ} \mathrm{C}$ was presented. In this case the average value of attenuation coefficient $\tilde{\gamma}_{\text {avg }}$ decreases with the heating time increase (Fig. 9c). However, the width of compartment changes of the attenuation coefficients has the lowest value after process of preliminary diffusion. The heating processes cause the essential growth of $\Delta \tilde{\gamma}$ (Fig. 9d).

\subsection{Refractive index profiles received during electrodiffusion process}

As it was presented in previous studies $[8,9]$ in the case of diffusion with the external electric field (the electrodiffusion process) there exists vast influence possibility on refractive index profile shape of the gradient waveguides 

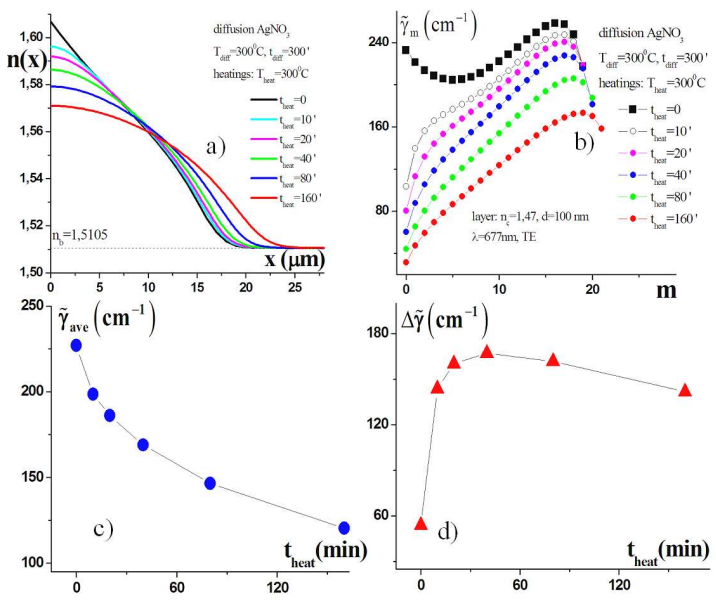

Fig. 9. Changes of modal attenuation characteristic as a result of heating process after diffusion $T_{\text {diff }}=300^{\circ} \mathrm{C}$, $t_{\text {diff }}=300^{\prime}:$ a) refractive index profiles, b) modal attenuation distributions, c) changes of average value of modal attenuation and d) partition spread in value changes of modal attenuation a result of heating processes.

received during such processes. Technological parameters which have the greatest impact on final shapes of refractive profiles are: possibility of direction changes of the electric field polarization during the process, value of electrical field intensity, and duration time of suitable polarizations. The final effects of proper selection among these parameters are refractive index profiles of convex, concave and linear shapes [9]. The influence of heating processes on modal attenuation distribution for planar waveguides received during the electrodiffusion process with fixed polarization was firstly analysed. In such processes the structures with convex shape of refractive profile can be made. Optical waveguides were produced during the processes conducted in temperature $T_{\text {ediff }}=300^{\circ} \mathrm{C}$, and time $t_{\text {ediff }}=1 \mathrm{~h}$. Two cases of electric field use with intensity $E=4.5 \mathrm{~V} / \mathrm{mm}$ as well as $E=27.3 \mathrm{~V} / \mathrm{mm}$ respectively, was analysed. The growth in applied intensity of electric field (at the same process temperature and the same time of its duration) inflicts that the refractive index profile of the optical waveguide becomes more convex (Fig. 10a and Fig. 11a). In Fig. 10a refractive index profile of the gradient waveguide received during electrodiffusion process with the intensity of electrical field $E=4.5 \mathrm{~V} / \mathrm{mm}$ and after heating $\left(T_{\text {heat }}=300^{\circ} \mathrm{C}, t_{\text {heat }}=15^{\prime}, 30^{\prime}, 60^{\prime}, 120^{\prime}\right.$, and $\left.240^{\prime}\right)$ were introduced. Also in this case the influence of heating process on shape of profile is clearly visible. Similarly to the diffusion process the growth in its depth and major decrease of refractive index value near the surface is observed. It is easy to notice that modal attenuation coefficient values will decrease with the increase of heating process duration (Fig. 10b). It should be pointed out that the average values of diffusion coefficient $\tilde{\gamma}_{\text {avg }}$ (Fig. 10c) as well as compartments of their changes $\Delta \tilde{\gamma}$ (Fig. 10d) will decrease with the increase of heating time.

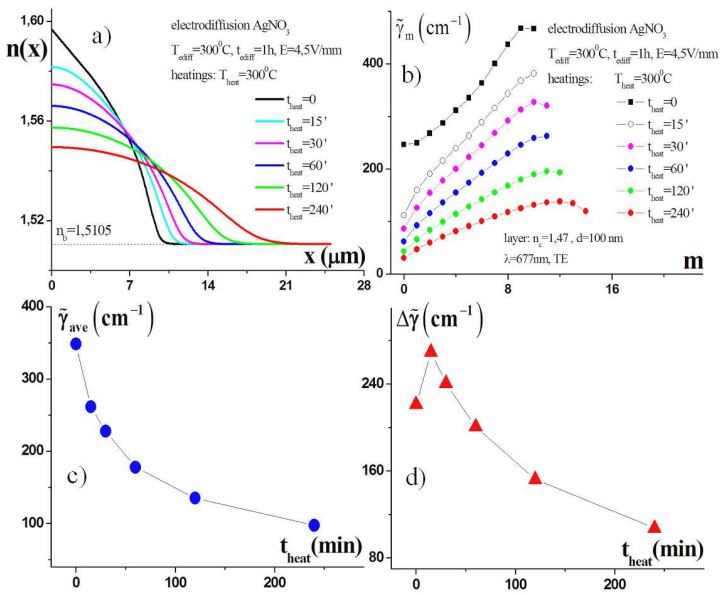

Fig. 10. Changes of modal attenuation characteristic as a result of heating process after electrodiffusion: $E=$ $4.5 \mathrm{~V} / \mathrm{mm}, T_{\text {ediff }}=300^{\circ} \mathrm{C}, t_{\text {ediff }}=1 \mathrm{~h}$. a) - d) as in Fig. 9.

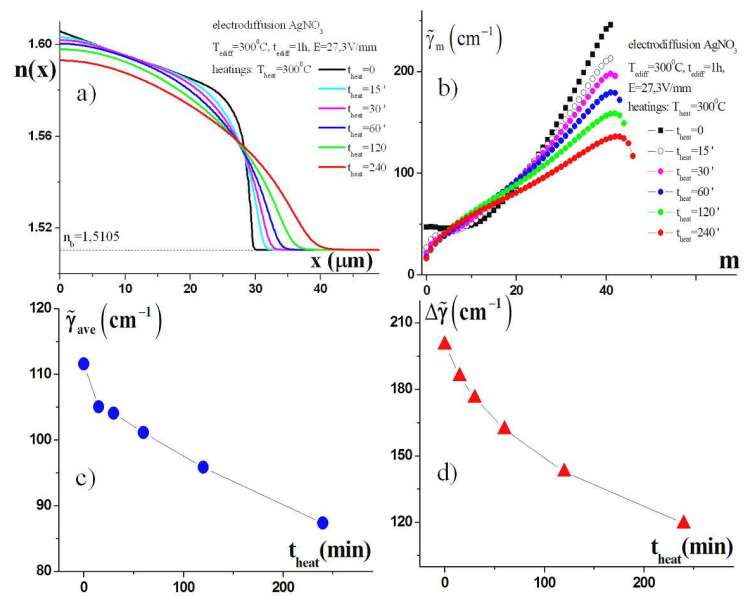

Fig. 11. Changes of modal attenuation characteristic as a result of heating process after electrodiffusion: $E=$ $27.3 \mathrm{~V} / \mathrm{mm}, T_{\text {ediff }}=300{ }^{\circ} \mathrm{C}, t_{\text {ediff }}=1 \mathrm{~h}$. a) $\left.-\mathrm{d}\right)$ as in Fig. 9.

For the electrodiffusion process realized in the same temperature $T_{\text {ediff }}=300^{\circ} \mathrm{C}$ and time $t_{\text {ediff }}=1 \mathrm{~h}$ when the intensity of electric field was set to $E=27.3 \mathrm{~V} / \mathrm{mm}$ the influence of the same heating processes on final shape of refractive index profile is now smaller (Fig. 11a). The large concentration of admixture ions $\left(\mathrm{Ag}^{+}\right.$ions) near the glass surface inflicts that the results of the same heating processes is smaller (insignificant change in refraction index values). Also the modal attenuation changes characteristics runs differently (Fig. 11b) (in some section they overlap to each other). The character of average attenuation coefficients' values changes $\tilde{\gamma}_{\text {avg }}$ (Fig. 11c) as well as compartments widths of the attenuation coefficient distribution changes $\Delta \tilde{\gamma}$ (Fig. 11d) in function of heating time 
is very similar. Waveguides with the concave shape of refractive index profile can be received in the electrodiffusion with change of polarization direction during the process. The influence of heating processes on refractive index profile of the waveguide produced in electrodiffusion process with the following parameters: $T_{\text {ediff }}=298^{\circ} \mathrm{C}$, $E=+4.5 \mathrm{~V} / \mathrm{mm}, t=45^{\prime}, E=-18.2 \mathrm{~V} / \mathrm{mm}, t=45^{\prime}$, was introduced in Fig. 12a. The temperature of heating processes in this case was $T_{\text {heat }}=300^{\circ} \mathrm{C}$ and the heating times $t_{\text {heat }}=15^{\prime}, 30^{\prime}, 60^{\prime}, 120^{\prime}$ and $240^{\prime}$.
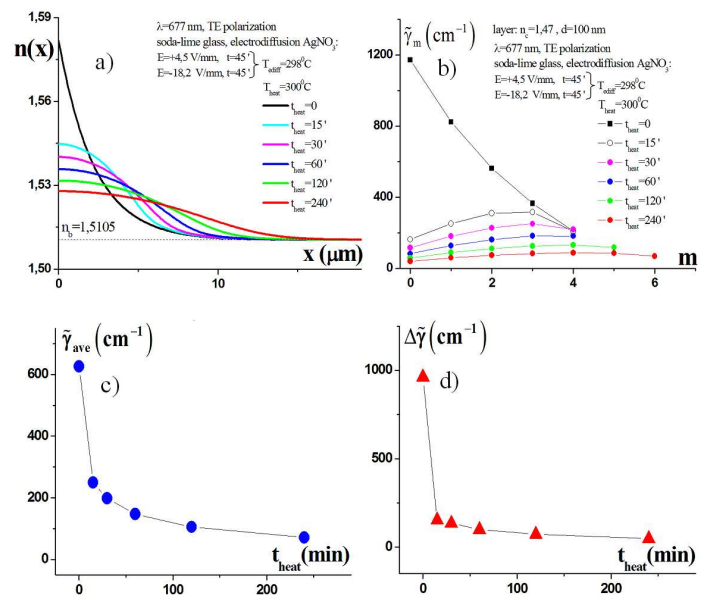

Fig. 12. Changes of modal attenuation characteristic as a result of heating process after electrodiffusion: $T_{\text {ediff }}=298^{\circ} \mathrm{C}, E=+4.5 \mathrm{~V} / \mathrm{mm}, t=45^{\prime}, E=$ $-18.2 \mathrm{~V} / \mathrm{mm}, t=45^{\prime}$. a) - d) as in Fig. 9 .

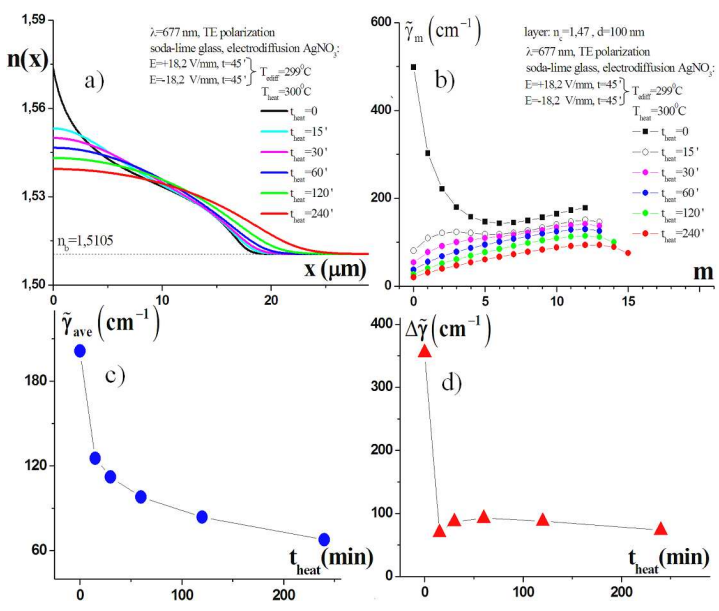

Fig. 13. Changes of modal attenuation characteristic as a result of heating process after electrodiffusion: $E=+18.2 \mathrm{~V} / \mathrm{mm}, t_{\text {ediff }}=45^{\prime}$ and $E=-18.2 \mathrm{~V} / \mathrm{mm}$, $t_{\text {ediff }}=45^{\prime}, T_{\text {ediff }}=299^{\circ} \mathrm{C}$. a) - d) as in Fig. 9 .

There is a clearly perceptible heating process influence on shape of refractive index profile (Fig. 12a). Heating processes strongly improve courses of modal attenuation (Fig. 12b). The growth of heating time causes significant reduction of average modal attenuation $\tilde{\gamma}_{\text {avg }}$ values

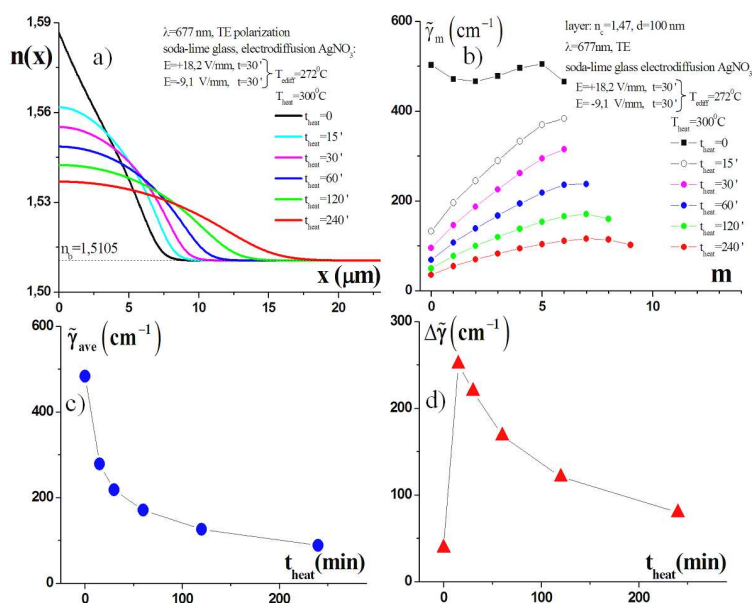

Fig. 14. Changes of modal attenuation characteristic as a result of heating process after electrodiffusion: $E=$ $+18.2 \mathrm{~V} / \mathrm{mm}, t_{\text {ediff }}=30^{\prime}$ and $E=-9.1 \mathrm{~V} / \mathrm{mm}, t_{\text {ediff }}=$ $30^{\prime}, T_{\text {ediff }}=272^{\circ} \mathrm{C}$. a) - d) as in Fig. 9 .

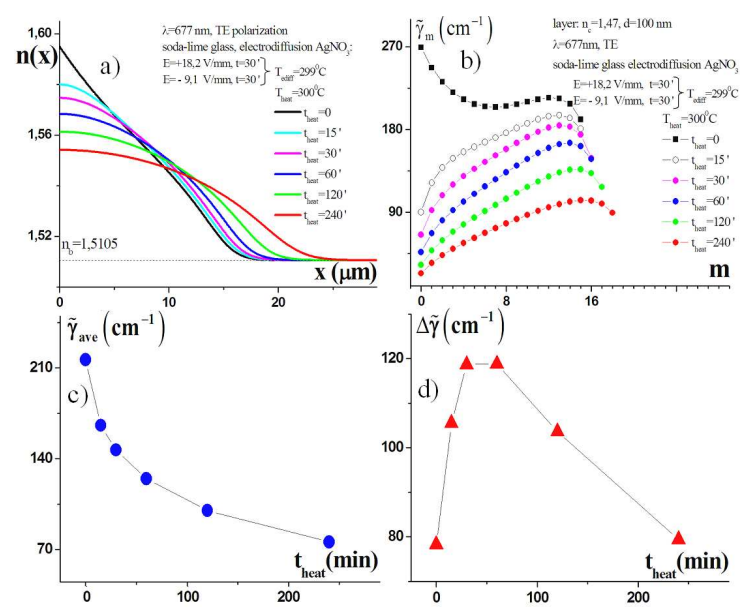

Fig. 15. Changes of modal attenuation characteristic as a result of heating process after electrodiffusion: $E=$ $+18.2 \mathrm{~V} / \mathrm{mm}, t_{\text {ediff }}=30^{\prime}$ and $E=-9.1 \mathrm{~V} / \mathrm{mm}, t_{\text {ediff }}=$ $30^{\prime}, T_{\text {ediff }}=299^{\circ} \mathrm{C}$. a) - d) as in Fig. 9 .

(Fig. 12c). Range of their compartments changes $\Delta \tilde{\gamma}$ with growth of heating time will also narrow (Fig. 12d).

Figure 13a represents the effects of changes in refractive index profile shape received as a result of electrodiffusion process in which the electric field intensity in both directions was equal to $E=+18.2 \mathrm{~V} / \mathrm{mm}, t_{\text {ediff }}=45^{\prime}$ as well as $E=-18.2 \mathrm{~V} / \mathrm{mm}, t_{\text {ediff }}=45^{\prime}$ and $T_{\text {ediff }}=299^{\circ} \mathrm{C}$ — subjected then to the heating processes. Simulations of heating processes were conducted in approximate temperature $T_{\text {heat }}=300^{\circ} \mathrm{C}$. The modal attenuation distribution corresponding to refractive index profiles from Fig. 13a was introduced in Fig. 13b. In this case similarly as before the fall of average modal attenuation coefficient $\tilde{\gamma}_{\text {avg }}$ values is observed with the growth of heating time (Fig. 13c). Function of modal attenuation distribution changes $\Delta \tilde{\gamma}$ is presented in Fig. 13d. For heating times $t_{\text {heat }}=15^{\prime}$ as well as the $t_{\text {heat }}=240^{\prime}$ the compartment 
changes achieve the smallest values. In other cases where the heating process was applied these values are higher.

In Figs. 14 and 15 the influence of heating processes on modal attenuation distribution in waveguides which have nearly linear refractive index profiles is presented. Such profiles characterize planar structures produced in electrodiffusion processes with the following values of electric field intensities and times of their application: $E=$ $+18.2 \mathrm{~V} / \mathrm{mm}, t_{\text {ediff }}=30^{\prime}$ as well as $E=-9.1 \mathrm{~V} / \mathrm{mm}$, $t_{\text {ediff }}=30^{\prime}$. The temperature of electrodiffusion process in which waveguides shown in Figs. 14a and 15a were produced was $T_{\text {ediff }}=272^{\circ} \mathrm{C}$ and $T_{\text {ediff }}=299^{\circ} \mathrm{C}$ respectively. In both cases the simulations of heating processes were conducted in temperature $T_{\text {heat }}=300^{\circ} \mathrm{C}$. Heating times were: $t_{\text {heat }}=15^{\prime}, 30^{\prime}, 60^{\prime}, 120^{\prime}$ and $240^{\prime}$. Modal attenuation distributions corresponding to refractive index profiles of the waveguides after heating processes are shown in Figs. 14b and 15b. With lack of heating process the modal attenuation compartment changes $\Delta \tilde{\gamma}$ have the smallest value (Figs. 14d and 15d). After short heating process $\Delta \tilde{\gamma}$ reaches maximum value, however with growth of heating time characteristics become flattened. For profiles received after diffusion process in temperature $T_{\text {diff }}=272^{\circ} \mathrm{C}$ modal attenuation coefficients achieve definitely higher values. The average values of modal attenuation $\tilde{\gamma}_{\text {avg }}$ in both cases are diminishing function of heating time (Figs. 14c and 15c).

\section{Summary and conclusions}

The results of the investigations introduced in this work show the influence of heating processes on modal attenuation distributions of waveguides with different shape of initial refraction index profile. Main goal was to show the utilization possibility of folded optical structures (the multimode gradient planar waveguide + the homogeneous sensor layer) in amplitude sensors.

Received results have theoretical character. They are based however on experimentally appointed thermal diffusion coefficients of the exchanged ions $\mathrm{Ag}^{+} \leftrightarrow \mathrm{Na}^{+}$in soda-lime glass (by Menzel-Glässer). This easily accessible glass is known for its good optical quality. The temperature dependences acquaintance of diffusion coefficients of exchanged ions for this glass was the basis for all calculations. Good compatibility of expected refractive index profiles in such glass with their measured equivalents were confirmed in previous investigations. Conducted calculations have therefore cognitive value.

Different shapes of the refraction index profiles received during diffusion processes, as well as its variation with assist of electric field - electrodiffusion, were considered. Results clearly show the tendency of changes and possible direction through future investigations. Pre- sented results allow us to draw out several essential conclusions. The use of heating process, which is required for drying absorptive layer deposited on gradient structure in every case causes changes in shape of refraction index profile received in diffusion process. Each time the presence of heating process caused decrease of the average value of attenuation coefficients. Promising results can be observed for concave and convex profiles, where with the increase of heating time the value of changes compartment of the attenuation coefficients gets clearly smaller.

Generally, there is no indication of a refractive index profile of the planar waveguide for which modal attenuation characteristic would be a constant function. Conducted calculations however show that for concave refractive index profiles, heating processes give the best flattening effect of modal attenuation characteristics.

\section{Acknowledgments}

The work was sponsored by the State Committee for Scientific Research (NCBiR) within the grant N R01 034 06/2009.

\section{References}

[1] G. Stewart, J. Norris, D.F. Clarc, B. Culshaw, Int. J. Optoelectron. 6, 227 (1991).

[2] P. Karasiński, Opto-Electron. Rev. 19, 10 (2011).

[3] P. Karasiński, R. Rogoziński, Opt. Commun. 269, 76 (2007).

[4] H. Gnewuch, H. Renner, Appl. Opt. 34, 1473 (1995).

[5] R. Rogoziński, Planar Waveguide Structures Produced by Ion Exchange in Glasses - Selected Aspects of Production Technology, Measurement of Optical Properties and Numerical Modelling of Structures, Monograph 135, Gliwice 2007 (in Polish).

[6] R. Rogoziński, Proc. SPIE 7120, 71200F (2008).

[7] R. Rogoziński, P. Karasiński, Opto-Electron. Rev. 13 , 229 (2005).

[8] R. Rogoziński, Opt. Appl. 34, 489 (2004).

[9] R. Rogoziński, Opt. Appl. 28, 331 (1998).

[10] P. Karasiński, R. Rogoziński, Proc. SPIE 5576, 181 (2004).

[11] P. Karasiński, Proc. SPIE 6608, 66080W (2007).

[12] C. Tyszkiewicz, P. Karasiński, R. Rogoziński, Acta Phys. Pol. A 118, 1262 (2010).

[13] A.W. Snyder, J.D. Love, Opical Waveguide Theory, Chapman and Hall, London 1983.

[14] E. Anemogiannis, E.N. Glytsis, J. Lightwave Technol. 10, 1344 (1992).

[15] C. Chen, P. Berini, D. Feng, S. Tanev, V.P. Tzolov, Opt. Expr. 7, 260 (2000). 